\title{
Electro-optic Coefficient Measurement of the Double Electro-Optic Molecules Polymer based on Terahertz Application
}

\author{
Zhang Ying ${ }^{1,3}$, Qiu Chengjun ${ }^{1}{ }^{*}$, Li You $^{2}$, Zhang Wenlong ${ }^{3}$ and Wang Xuan ${ }^{3}$ \\ ${ }^{1}$ Electronic Engineering College of Heilongjiang University, Harbin, 150080, China; \\ ${ }^{2}$ Harbin Research Institute of Electrical Instrument, Harbin, 150028, China; \\ ${ }^{3}$ State Key Laboratory Breeding Base of Dielectric Engineering, Harbin, 150080, \\ China \\ *waterunderbridge@163.com
}

\begin{abstract}
In the terahertz (THz) science research field, electro-optic (EO) polymer possesses many superior characters compared with mineral crystal. Two kinds of the side group EO polymer, polyphosphazene with plmaol grained by 2, 4-dinitroaniline and polyphenylacrylate containing p-nitro bisazo group, are synthesized and characterized. For higher EO coefficient, the double EO molecules polymer thin films are prepared by mixing a small nonlinear optical (NLO) molecule into the side group EO polymer. The principles of preparation are given and the EO coefficients are tested. The effect on the EO coefficients by different mixing proportion and different poling temperature are analyzed in details. Experiments results show that the double EO molecules polymer has fine stability, higher EO coefficient and can be easily processed. This kind of material has the potential to be widely applied in the emission and detection of THz radiation.
\end{abstract}

Keywords: THz science; mineral crystal; double EO molecules polymer; EO coefficient

\section{Introduction}

EO polymer has been the research focus in THz science field nowadays. Compared with mineral crystal, EO polymer possesses many superior characters. This kind of material is very stable against temperature, oil, radiation, solvents and so on ${ }^{[1,2]}$. Meanwhile, EO polymer has fast response time, good plasticity, simple device fabrication and good mechanical property. The most important thing is that this polymer possesses higher EO coefficient than other mineral crystal materials. Due to these unique functional properties, EO polymer have been developed and utilized in biomedicine, liquid crystals, aerospace, power electronics, energy storage and generation, membrane science, elastomers, surface science, photoelectric functional materials, military manufacturing and biomedical science ${ }^{[3,4,5]}$.

Although the EO coefficient of the EO polymer has been improved, it is still not enough to meet the requirement of scientific research. Two kinds of the side group EO polymer, polyphosphazene with plmaol grained by 2, 4-dinitroaniline and polyphenylacrylate containing p-nitro bisazo group, are synthesized and characterized ${ }^{[6,7]}$. The UV-visible absorption spectrum and the IR spectrum are obtained. The EO coefficients are tested by the simple reflection technique. For higher EO coefficient, the double EO molecules polymer thin films are prepared by mixing a small nonlinear optical (NLO) molecule into the side group EO polymer. The EO coefficients are tested. 


\section{Simple Reflection method}

Under the influence of electro-optic effect, the phase delay of the linearly polarized light happens. The interference of $\mathrm{p}$ light and $\mathrm{s}$ light can reflect the EO coefficient of the polymer. This method is named the simple reflection technique ${ }^{[8]}$. The schematic of the method is shown in Fig.1.

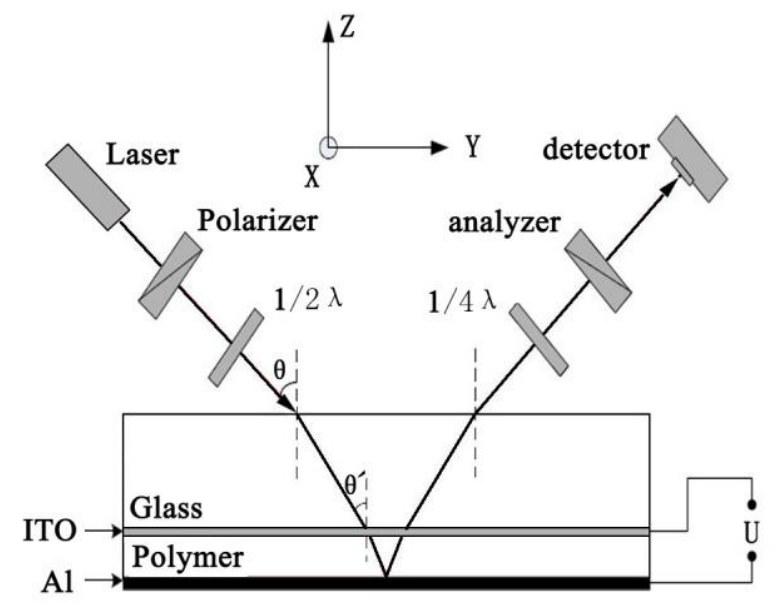

Figure 1 The Schematic of the Method

Incident light is converted to the linearly polarized light under the action of the polarizer. Than the linearly polarized light reaches the glass surface with the incidence angle of $\theta$ and refracts twice at the glass surface and the ITO respectively. Next, the light reaches the surface of the polymer with the incidence angle of $\theta$ '. Under the influence of electro-optic effect, birefringence effect of the p light and s light happen that is reflected by the $\mathrm{Al}$ electrode. Than the $\mathrm{p}$ light and $\mathrm{s}$ light propagate though the $1 / 4$ slide, and refract with each other at the analyzer. The results are tested by the detector and the lock-in amplifier.

The light intensity at the detector is given by:

$$
I_{\text {out }}=2 I_{c} \sin ^{2} \frac{\varphi_{s p}}{2}
$$

$2 I_{C}$ is the maximum value of the output light intensity, and $\varphi_{\mathrm{sp}}$ is the phase displacement. Suppose $\gamma_{33} \approx 3 \gamma_{31}$, than the coefficient can be given by:

$$
\gamma_{33}=\frac{3 \lambda I_{\mathrm{m}}}{4 \pi V_{\mathrm{m}} I_{\mathrm{c}} n^{2}} \cdot \frac{\left(n^{2}-\sin ^{2} \theta\right)^{3 / 2}}{\left(n^{2}-2 \sin ^{2} \theta\right) \sin ^{2} \theta}
$$

The $\lambda$ is the wavelength of the laser and $n$ is the refractive index of the polymer. The $V_{m}$ is the peak value of the voltage and $I_{m o}$ is the light intensity. 


\section{Side Group EO Polymer}

\subsection{Synthesis of the Material}

The diazonium salt solvent was made from p-nitro azo aniline, NaNO2, hydrochloric acid, and water. The polyacrylic acid benzyl ester, THF, $\mathrm{NaOH}$, distilled water, sodium dodecyl benzene sulfonate are stirred together to form the yellow solvent. Than the yellow solution react with the diazonium salt solution with the temperature of $0-4^{\circ} \mathrm{C}$ and the $\mathrm{PH}$ value of 5-7. The polyphenylacrylate containing p-nitro bisazo group can be obtained by drying the reaction product. The molecular formula is shown in Figure 2.

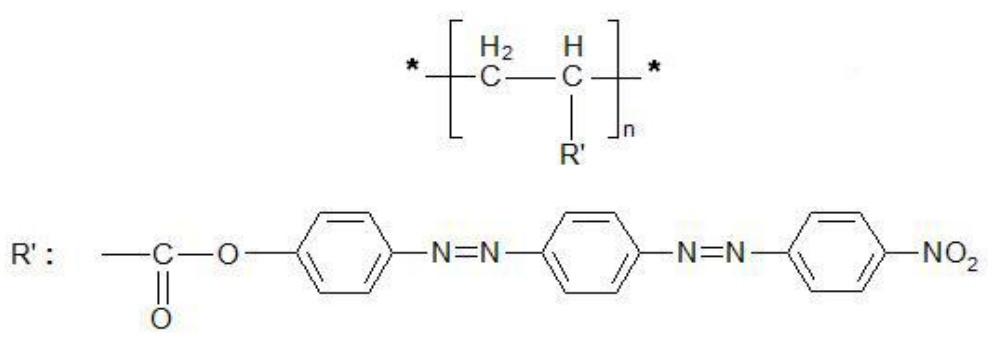

\section{Figure 2 The Molecular Formula of the Polyphenylacrylate Containing p-nitro Bisazo Group}

The phenol polyphosphazene, 2, 4-dinitroaniline, sodium nitrite, potassium bisulfate and distilled water are placed together to react for a period of time. Than the polyphosphazene with plmaol grained by 2, 4-dinitroaniline can be obtained by washing and drying the dark red reaction product. The molecular formula is shown in Fig.3.

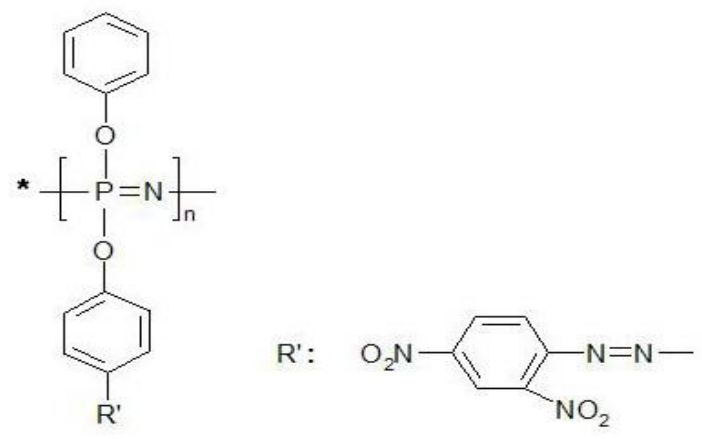

Figure 3 The Molecular Formula of the Polyphosphazene with plmaol Grained by 2, 4-dinitroaniline

\subsection{Structure Characterization}

The UV-visible absorption spectrum of the polyphenylacrylate containing p-nitro bisazo group is shown in Fig.4. The peak appeared at $425 \mathrm{~nm}$ to $475 \mathrm{~nm}$ is the absorption peak of azo group, which are the result of the $n-\pi^{*}$ transition. The result indicates that the azo group has been grafted to the main chain successfully. The IR spectrum of the polyphenylacrylate containing p-nitro bisazo group is shown in Fig.5. The peaks obtained at $1330 \mathrm{~cm}-1$ and 1588 $\mathrm{cm}-1$ are the characteristic absorption peaks of the $\mathrm{C}-\mathrm{O}$ and $\mathrm{N}=\mathrm{N}$. The absorption peak of the 
benzene p-substituted appears at $845 \mathrm{~cm}-1$. These absorption peaks observed indicate that the reaction product is the polyphenylacrylate containing p-nitro bisazo group really.

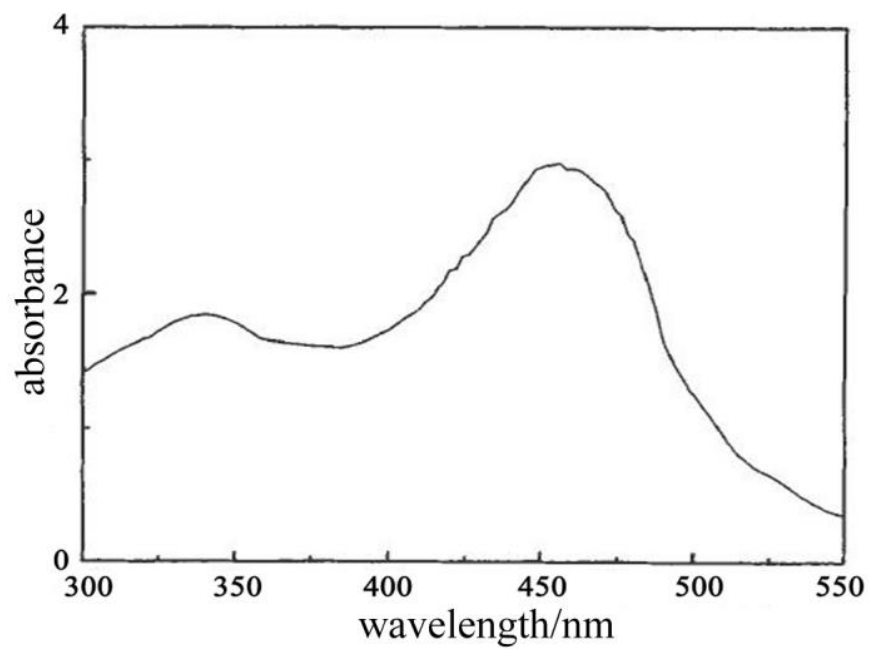

Figure 4 UV-visible Absorption Spectrum

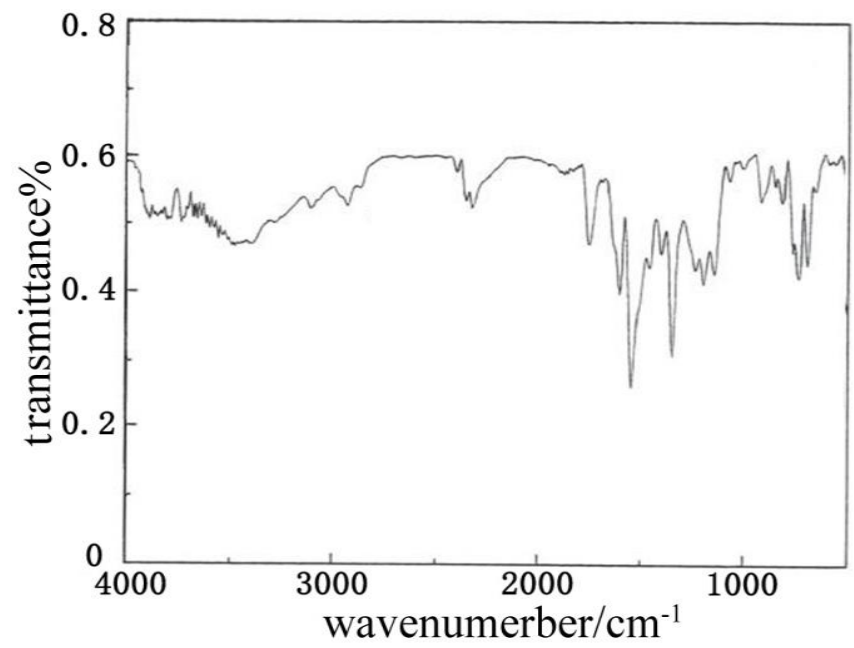

Figure 5 The IR Spectrum

The UV-visible absorption spectrum of the polyphosphazene with plmaol grained by 2, 4dinitroaniline is shown in Fig.6. The peak appeared at $400 \mathrm{~nm}$ to $475 \mathrm{~nm}$ is the absorption peak of azo group $(\mathrm{N}=\mathrm{N})$, which are the result of the $n-\pi^{*}$ transition. This indicates that the azo group has been grafted to the phenol polyphosphazene successfully. The IR spectrum of the polyphosphazene with plmaol grained by 2, 4-dinitroaniline is shown in Fig.7. The peaks obtained at $1050 \mathrm{~cm}-1$ and $927 \mathrm{~cm}-1$ are the characteristic absorption peaks of the P-O-C. The peaks obtained at $1195 \mathrm{~cm}-1$ and $1450 \mathrm{~cm}-1$ are the stretch absorption peaks of the $\mathrm{P}=\mathrm{N}$ and $\mathrm{C}=\mathrm{C}$. The benzene p-substituted peak appears at $855 \mathrm{~cm}-1$ and the benzene characteristic absorption peak appears at $731 \mathrm{~cm}-1$. These results observed indicate that the reaction product is the polyphosphazene with plmaol grained by 2 , 4-dinitroaniline really. 


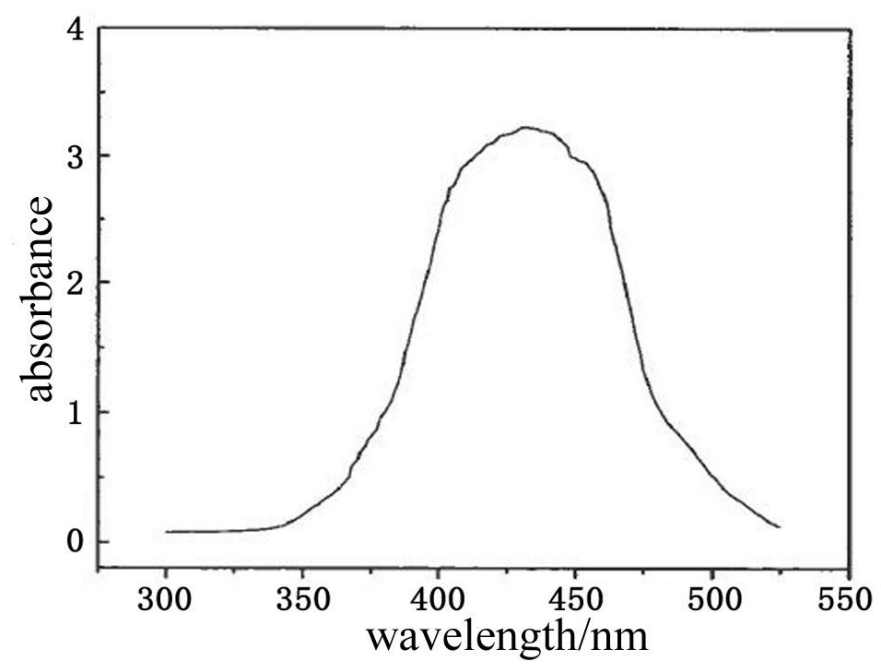

Figure 6 UV-Visible Absorption Spectrum

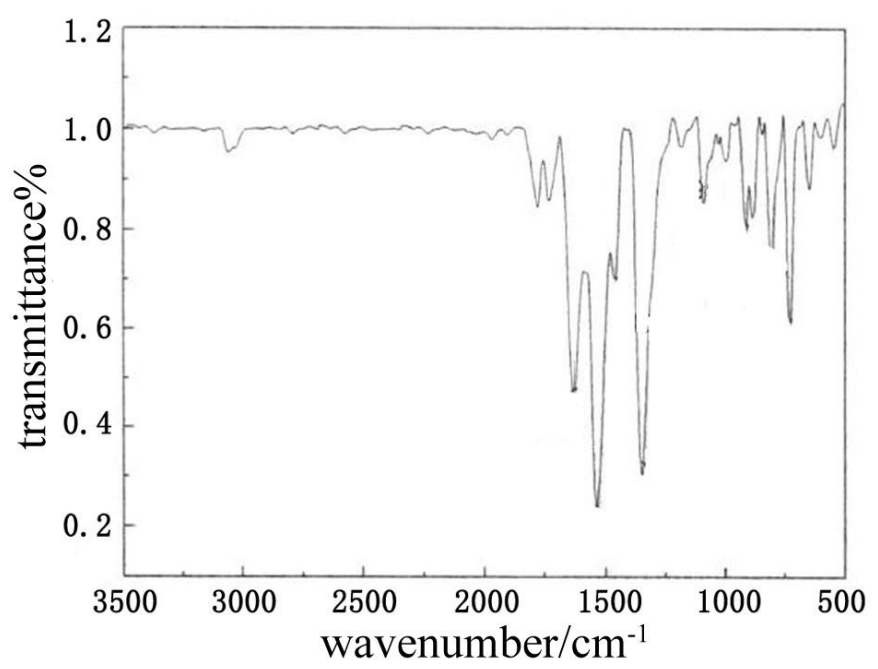

Figure 7. The IR Spectrum

\subsection{Measurement of the EO Coefficient}

The EO coefficients of the two side group EO polymers are tested by simple reflection method. The EO coefficient of the polyphenylacrylate containing p-nitro bisazo group is $12.36 \mathrm{pm} / \mathrm{V}$ and the EO coefficient of the polyphosphazene with plmaol grained by 2, 4dinitroaniline is $13.64 \mathrm{pm} / \mathrm{V}$.

\section{Double EO Molecules Polymer}

\subsection{Physical Mixture of the Small EO molecule}

Based on the side group EO polymer, double electro- optic molecules polymer can be obtained through small EO molecule physical doping for higher EO coefficient. Ethyl 4dimethylaminobenzoate (EDB) has been chosen as a small EO molecule for the experiment to improve the EO coefficient. The molecular formula of EDB is shown in Figure 8. 
<smiles>CCOC(=O)c1ccc(N(C)C)cc1</smiles>

\section{Figure 8 The Molecular Formula of EDB}

\subsection{The Effect on the EO Coefficients by Different Mixing Proportion}

In order to know the effect on the EO coefficients by different mixing proportion, nine groups of EDB with different qualities are mixed into the two kinds of the double EO molecules polymers respectively. The polyphenylacrylate containing p-nitro bisazo group is chosen as sample A and the polyphosphazene with plmaol grained by 2, 4-dinitroaniline is chosen as sample B. The qualities of EDB are $2 \mathrm{wt} \% 、 3 \mathrm{wt} \% 、 4 \mathrm{wt} \% 、 5 \mathrm{wt} \% 、 6 \mathrm{wt} \% 、 7 \mathrm{wt} \%$ 、 $8 \mathrm{wt} \% 、 9 \mathrm{wt} \% 、 10 \mathrm{wt} \%$. Than the EO coefficients of all the samples are tested. The results are shown in Fig.9.

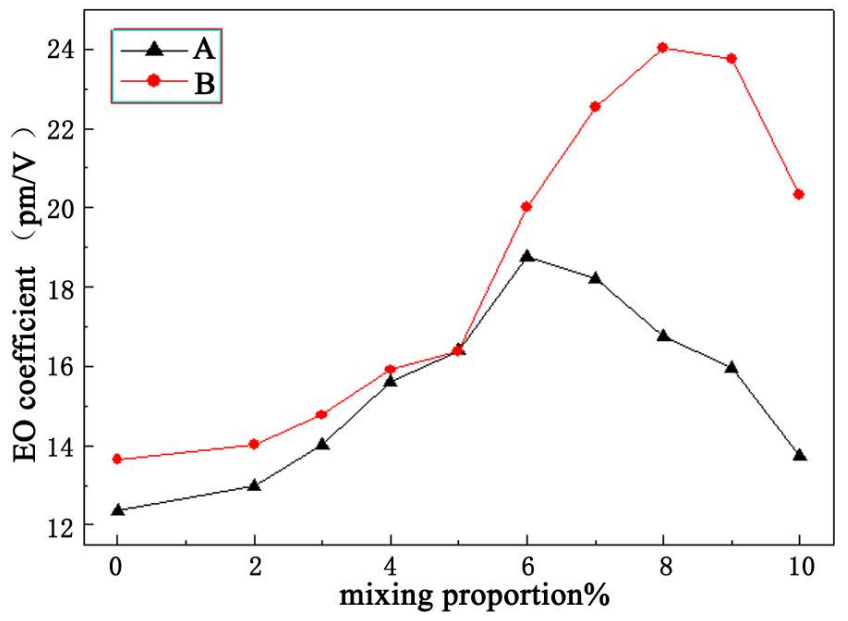

Figure 9 The Effect on the EO Coefficients by Different Mixing Proportion

For the polyphenylacrylate containing p-nitro bisazo group, the EO coefficient increases as the mixing proportion becomes larger. When the mixing proportion is $8 \mathrm{wt} \%$, the EO coefficient reaches its maximum value. Then the EO coefficient decreases as the mixing proportion becomes larger. The variation tendency of the polyphosphazene with plmaol grained by 2, 4-dinitroaniline is the same as the polyphenylacrylate containing p-nitro bisazo group. The only difference is that the EO coefficient reaches its maximum value when the mixing proportion is $6 \mathrm{wt} \%$. All the experimental results show that physical mixture of the small EO molecule can indeed improve the EO coefficient, although the effects are different for different materials. The decrease of the EO coefficient is due to the high mixing proportion, which leads to the dropping of the glass transition temperature. 


\subsection{The effect on the EO coefficients by different poling temperature}

Poling is that the chromophores in the EO polymer orient according to the direction of the external economy electric field ${ }^{[9,10]}$. In order to know the effect on the EO coefficients by different poling temperature, the EO coefficients of the two samples with suitable mixing proportion are tested respectively. The poling temperatures are $65^{\circ} \mathrm{C}$ and $75^{\circ} \mathrm{C}$. The results are shown in Fig.10 and Fig.11.

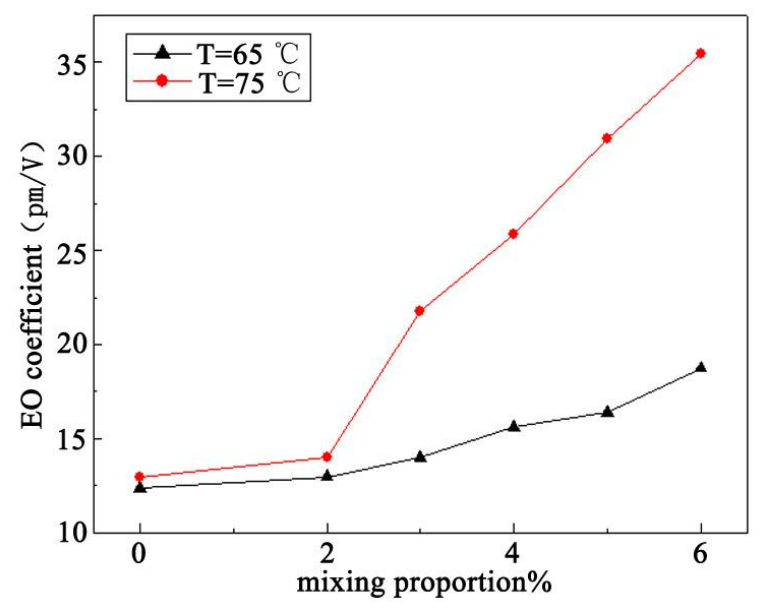

Figure 10 The Result for Sample A

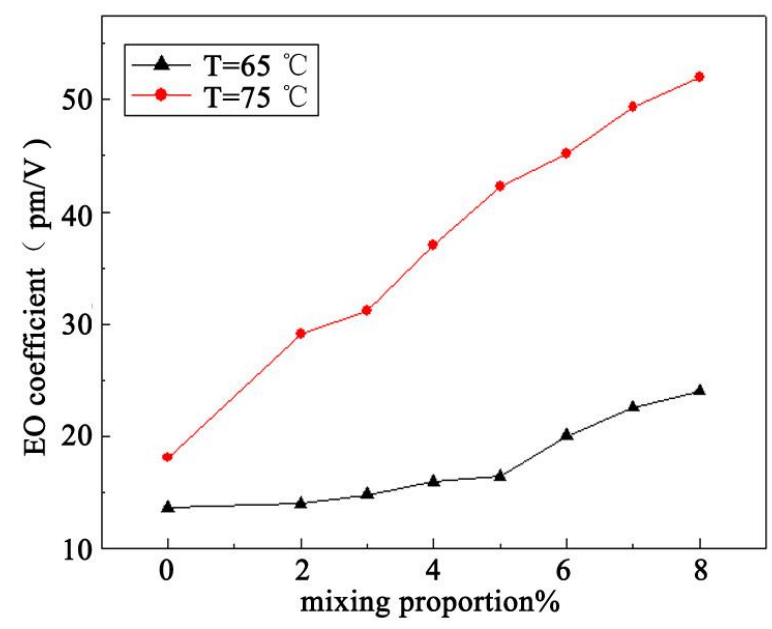

Figure 11 The Result for Sample B

\section{Conclusions}

The experimental results show that the EO coefficients can be improved by small EO molecule physical doping, while the maximum values are got at different mixing proportions for different polymers. For the polyphenylacrylate containing p-nitro bisazo group, the EO coefficient is improved by $51.7 \%$. For the polyphosphazene with plmaol grained by $2,4-$ dinitroaniline, the EO coefficient is improved by $76.1 \%$. Especially, the maximum value for the polyphosphazene with plmaol grained by 2, 4-dinitroaniline can be $24.02 \mathrm{pm} / \mathrm{V}$. In addition, the poling temperature can affect the EO coefficients too. The EO coefficient 
increases as the poling temperature becomes larger. The poling temperature must be around the glass transition temperature, the closer the batter.

In conclusion, the double EO molecules polymer has fine stability, higher EO coefficient and can be easily processed. This kind of material has the potential to be widely applied in the emission and detection of $\mathrm{THz}$ radiation.

\section{Acknowledgements}

The work is supported by the Natural Foundation of Heilongjiang Province (E201445) and the Research Foundation of Education Bureau of Heilongjiang Province (12541183, 12511078).

\section{References}

[1] M. Eralp, J. Thomas and S. Tay, "High-performance photorefractive polymer operating at 975nm", [J], Applied physics letters, vol. 85, no. 7, (2004), pp. 1095-1101.

[2] J. Qiang, W. Xuan and L. Zhiyuan, "Measurement of the Electro-Optic Coefficients in Electro-Optic Polymer Thin Film Devices with Interdigitated Coplanar Electrodes", [J], ACTA OPTICA SINICA, vol. 33, no. 9, (2013), pp. 0931001.

[3] M. X. He and T. Chen, "Application of terahertz science and technology in biology and medicine research", [J], Journal of Electronic Measurement and Instrument, vol. 26, no. 6, (2012), pp. 471-483.

[4] H. S. Peter, "Terahertz technology", [J], IEEE Transactions, vol. 50, no. 3, (2002), pp. 910-928.

[5] B. G. Zhang, J. Q. Yao and H. Zhang, et al., "Angle-tuned signal-resonated optical parametric oscillator based on periodically poled lithium niobate", [J], Chinese Optic Letters, vol. 1, no. 6, (2003), pp. 346-349.

[6] Z. Ying, Q. Chengjun and J. Qiang, et al., "Electro-optic sensor based on side group polymer and Terahertz wave detecting system", [J], Chinese Journal of Scientific Instrument, vol. 34, no. 13, (2013), pp. 28242830.

[7] Y. Wenwen and F. Huakang, et al., "Preparation and relaxation behavior of layered double hydroxide modified PU/PMMA nanocomposites", [J], Acta Polymerica Sinica, vol. 9, (2013), pp. 1212-1218.

[8] S. Han and J. W. Wu, "Single-beam polarization interferometry measurement of the linear electro-optic effect in poled polymer films with a reflection configuration", [J], Optical Society of America, vol. 14, no. 5, (1997), pp. 1131-1137.

[9] S. Prashant and G. M. Singh, "Thermally stimulated depolarization current and dielectric relaxation spectroscopy in poly (vinylidene fluoride) samples", [J], Iranian Polymer Journal, vol. 17, no. 3, (2008), pp. 183-190.

[10] J. A. Giacometti, “Constant Current Corona Triode with Grid Voltage Control”, [J]. Review of Scientific Instrume, vol. 161, (1990), pp. 1143-1150. 